\title{
Enumeration of $(16,4,16,4)$ Relative Difference Sets
}

\author{
David Clark \\ Department of Mathematics \\ University of Minnesota \\ Minneapolis, MN 55455, USA \\ dcclark@umn.edu
}

\author{
Vladimir D. Tonchev* \\ Department of Mathematics \\ Michigan Technological University \\ Houghton, MI 49931, USA \\ tonchev@mtu.edu
}

Submitted: Oct 5, 2012; Accepted: Mar 21, 2013; Published: Mar 31, 2013

\begin{abstract}
A complete enumeration of relative difference sets (RDS) with parameters $(16,4,16,4)$ in a group of order 64 with a normal subgroup $N$ of order 4 is given. If $N=Z_{4}$, three of the eleven abelian groups of order 64 , and 23 of the 256 nonabelian groups of order 64 contain $(16,4,16,4)$ RDSs. If $N=Z_{2} \times Z_{2}$, six of the abelian groups and 194 of the non-abelian groups of order 64 contain $(16,4,16,4)$ RDSs.
\end{abstract}

Keywords: Relative difference set; symmetric net.

\section{Introduction}

A relative difference set (RDS) with parameters $(m, n, k, \lambda)$ in a finite group $G$ of order $m n$ relative to a normal subgroup $N$ of order $n$ is a $k$-subset $R$ of $G$ such that every element of $g \in G \backslash N$ appears exactly $\lambda$ times in the multiset $S=\left\{a b^{-1} \mid a, b \in R, a \neq b\right\}$, and no element of $N$ appears in $S$ [1]. An RDS is called abelian if $G$ is abelian, and nonabelian otherwise.

Relative difference sets are closely related to difference sets, group-divisible designs, generalized Hadamard matrices, symmetric nets, and finite geometry [1], [4], [6]. A comprehensive survey on RDS is the paper by Pott [5]. The existence problem of $\left(p^{a}, p^{b}, p^{a}, p^{a-b}\right)$ RDSs is considered to be one of the most important questions concerning RDSs [5].

In [7], Schmidt studied the existence of abelian $\left(p^{a}, p^{b}, p^{a}, p^{a-b}\right)$ RDS, and settled the existence problem of abelian $(16,4,16,4)$ RDS completely.

\footnotetext{
${ }^{*}$ The authors acknowledge support by NSA Grant H98230-12-0213.
} 
In this paper, a complete enumeration of $(16,4,16,4)$ RDSs is given, for all groups, abelian and nonabelian, of order 64 . In summary, RDSs exist in 6 of the 11 abelian groups of order 64, as well as in 195 of the 256 nonabelian groups of order 64 . If $N=Z_{4}$, three of the eleven abelian groups of order 64 , and 23 of the 256 non-abelian groups of order 64 contain $(16,4,16,4)$ RDSs. If $N=Z_{2} \times Z_{2}$, six of the abelian groups and 194 of the non-abelian groups of order 64 contain $(16,4,16,4)$ RDSs. The computer algebra package Magma [2] was used in the computations.

\section{$2 \quad$ RDS and symmetric nets}

Our approach to the enumeration of $(16,4,16,4)$ RDSs is based on their link to incidence structures known as symmetric $(4,4)$-nets.

A symmetric $(4,4)-n e t^{1}$ is an incidence structure $I=(X, \mathcal{B})$ consisting of a set $X$ of 64 points and a collection $\mathcal{B}$ of 64 blocks, each block being a subset of 16 points of $X$, having the following properties:

- Each point belongs to 16 blocks.

- There exists a partition $\mathcal{P}$ of the point set $X$ into 16 subsets of size 4, called groups, so that every two points belonging to different groups appear together in exactly 4 blocks, while every two points belonging to the same group do not appear together in any block.

- The 64 blocks are partitioned into 16 parallel classes, each class consisting of 4 pairwise disjoint blocks, so that every two blocks belonging to different parallel classes share exactly 4 points.

Other terms used for a structure with the above properties are group-divisible design, or a transversal design [1].

An automorphism of an incidence structure $I$ is any permutation of the point set which preserves the collection of blocks. The set of all automorphisms of $I$ form a group, called the full automorphism group, Aut(I), of $I$. The subgroups of $A u t(I)$ are called automorphism groups.

A symmetric $(4,4)$-net is class-regular if it admits an automorphism group $N$ of order 4 which acts transitively on each group of points and each parallel class of blocks. The group $N$ is then called a group of bitranslations.

If $R$ is a $(16,4,16,4)$ RDS in a group $G$ of order 64 , relative to a normal subgroup $N \leqslant G$ of order 4 , one can associate with $R$ a class-regular $(4,4)$-net $I$ with point set $G$ and blocks being the subsets $B_{g} \subseteq G$ of the form

$$
B_{g}=\{R g \mid g \in G\}
$$

\footnotetext{
${ }^{1}$ More generally, a net is defined as a resolvable 1-design, and a symmetric net is a net with equal number of points and blocks. For more definitions concerning designs see [1].
} 
The partition $\mathcal{P}$ of the points into subsets of size 4 is defined as the partition of $G$ into cosets of $N$. Consequently, $G$ acts as an automorphism group of $I$, and the subgroup $N$ acts transitively on each point group and each parallel class.

Thus, any $(16,4,16,4) \mathrm{RDS}$ corresponds to a class-regular symmetric $(4,4)$-net which admits a regular automorphism group.

All nonisomorphic class-regular symmetric $(4,4)$-nets were enumerated by Harada, Lam and Tonchev in [4], and, implicitly, by Gibbons and Mathon in [3] (two incidence structures are isomorphic if there is an incidence preserving bijection between their point sets). Up to isomorphism, there are exactly 226 nets with group of bitranslations $N=$ $Z_{2} \times Z_{2}$, and 13 nets with $N=Z_{4}$.

These results reduce the enumeration of $(16,4,16,4)$ RDSs to finding sharply transitive regular subgroups $G$ of the full automorphism groups of those class-regular symmetric $(4,4)$-nets which admit automorphism groups acting transitively on the points, such that $N$ is a normal subgroup of $G$. We used Magma to find the conjugacy classes of sharply transitive regular subgroups.

There are 267 groups of order 64, of which 11 are abelian and 256 are nonabelian. Among the 226 nets with group of bitranslations $Z_{2} \times Z_{2}$, only 200 nonisomorphic regular subgroups of order 64 appeared within the automorphism groups of those nets, of which 6 were abelian and 194 were nonabelian. Among the 13 nets with group of bitranslations $Z_{4}$, only 26 nonisomorphic regular subgroups of order 64 appeared within the automorphism groups of those nets, of which 3 were abelian and 23 were nonabelian.

\section{The results}

Tables 1 and 2 list the nets with automorphism groups which admit regular subgroups with normal subgroup $N$. Each entry is as follows:

- \#: The index of the net within the list of nets with a group of bitranslations $N=\mathbb{Z}_{2} \times \mathbb{Z}_{2}$ available at http://www.math.mtu.edu/ tonchev/Z2Z2nets and at http://www.math.mtu.edu/ tonchev/Z4nets for the nets with $N=\mathbb{Z}_{4}$. Missing indices indicate that the corresponding nets do not have transitive automorphism groups.

- Order: The order of the automorphism group.

- 2-Rank: The 2-rank of the incidence matrix of the net.

- Total: In the format $x / y, x$ indicates the total number of conjugacy classes of regular subgroups containing the group of bitranslations, found within the automorphism group of each net, while $y$ is the number of nonisomorphic regular subgroups.

- Abelian and Nonabelian: In the format $x / y, x$ indicates the number of conjugacy classes of each type of subgroup found within the net's automorphism group, while $y$ is the number of nonisomorphic subgroups. 
- List of indices: Below the previous data is a list of the indices of the regular subgroups of order 64 found in each automorphism group according to Magma's list of the groups of order 64 . Entries of the form $x(y)$ indicate that groups isomorphic to group $x$ appeared in $y$ distinct conjugacy classes. Entries marked with an asterisk are abelian, and all others are nonabelian.

Tables 3 and 4 give details about the structure of the regular abelian subgroups of order 64 found. Only such subgroups containing the relevant group $N$ of bitranslations were considered. Groups with the following indices had a single regular abelian subgroup isomorphic to $\mathbb{Z}_{2}^{6}$, and are not listed in Table 3: 6, 99, 100, 103, 104, 105, 107, 111, 113, $120,121,127,128,131,142,143,144,145,146,147,148,149,150,151,181,182,192$, 193, 194, 195, 196, 197, 198, 212, 214, 221, 224, 225, 226.

Tables 5 and 6 summarize the structures of the regular abelian subgroups of order 64 found within the nets.

Table 7 gives the structures of all abelian groups of order 64 which do not appear as a regular subgroup in any net. Consequently, those are the groups which do not support any $(16,4,16,4) \mathrm{RDS}$.

Table 1: $(16,4,16,4)$ RDS with $N=\mathbb{Z}_{2} \times \mathbb{Z}_{2}$

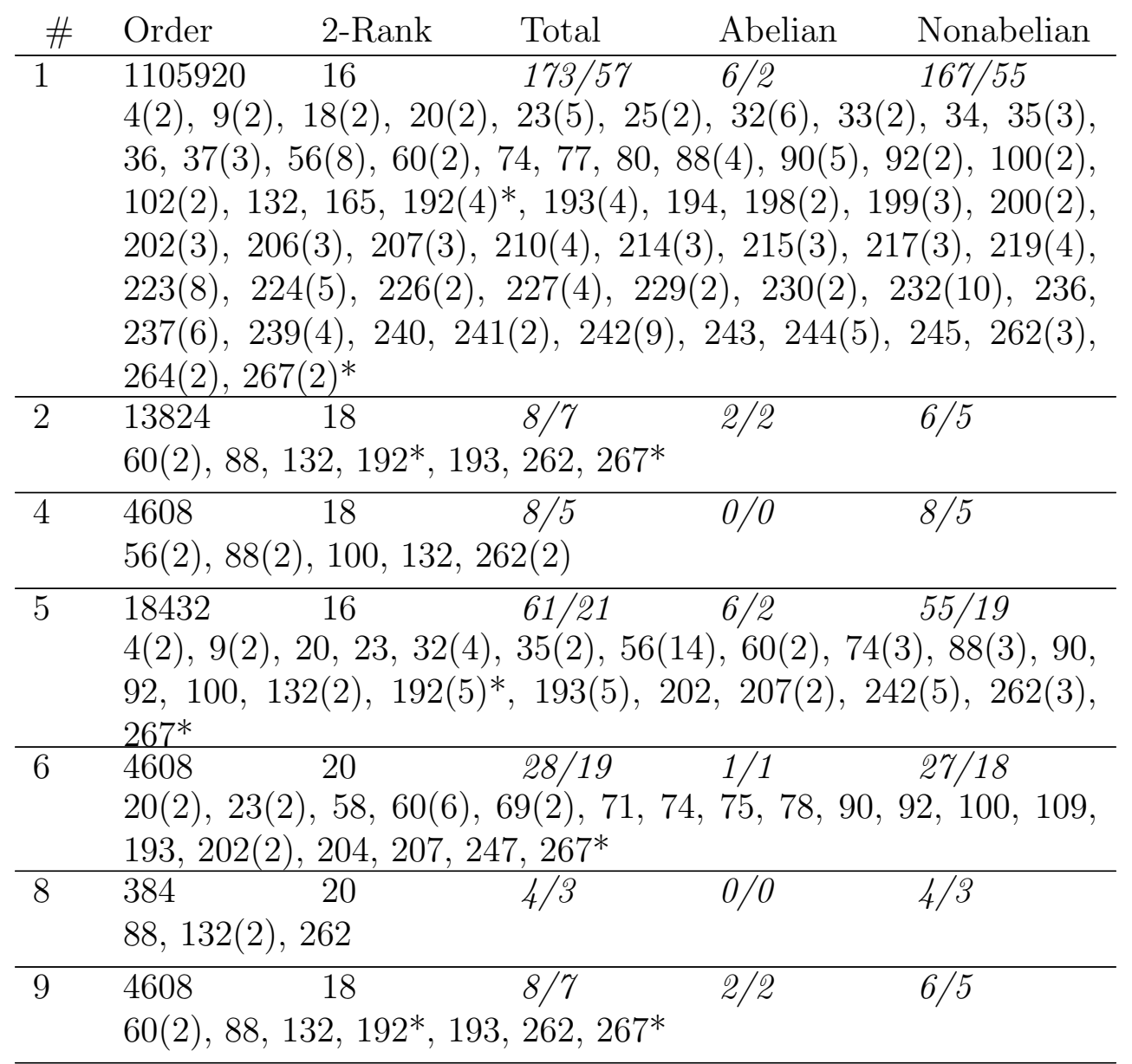


Table 1: $(16,4,16,4)$ RDS with $N=\mathbb{Z}_{2} \times \mathbb{Z}_{2}$

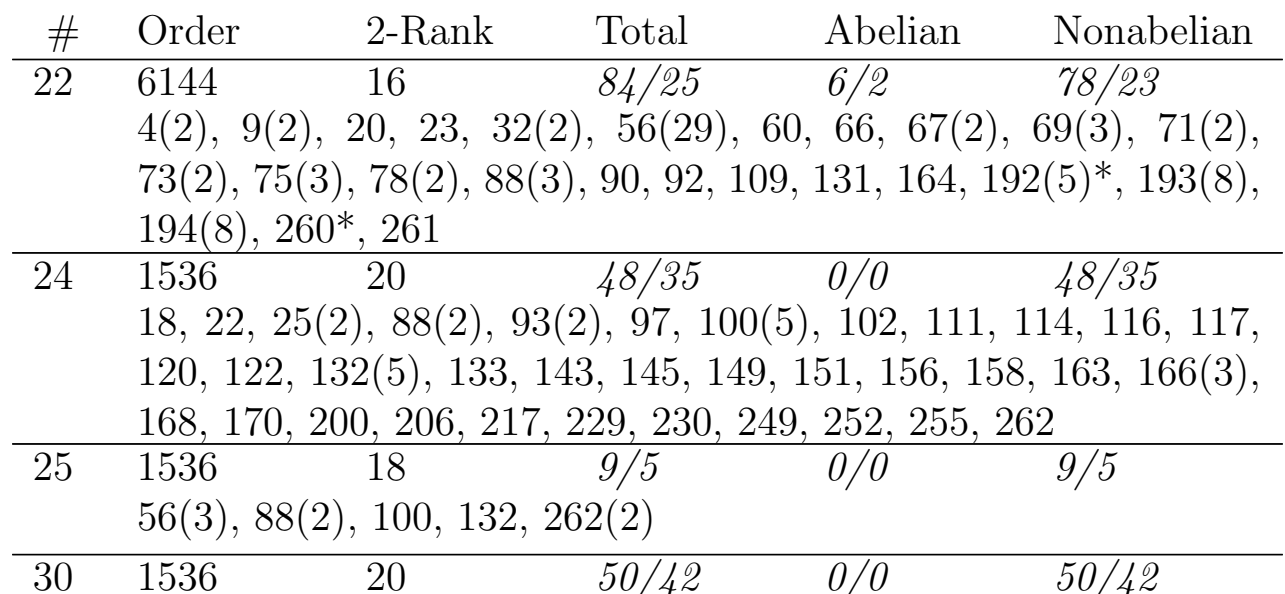

18, 22, 25(2), 33, 36, 91, 93(2), 97(2), 99, 100(2), 105, 111, 119, $120,121,122,129,130,131,132(2), 133,142,143,144,145(3)$, 146, 148, 149, 151, 156, 158, 166(2), 168, 170, 176, 178, 200, $249,251,252,254,255$

\begin{tabular}{|c|c|}
\hline 31 & 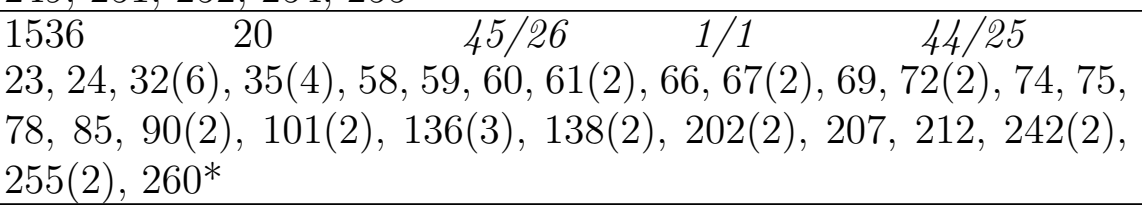 \\
\hline 32 & $\begin{array}{lc}128 & 20 \\
146,148,206,246^{*}\end{array}$ \\
\hline 33 & 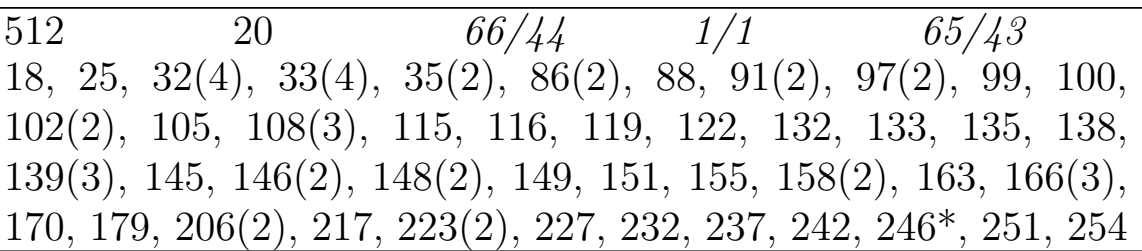 \\
\hline 36 & $\begin{array}{l}73728 \quad 160158 / 85 \quad 6 / 3 \quad 1152 / 82 \\
4(2), 9(2), 18(6), 20(6), 23(13), 25(6), 32(14), 33(10), 34(5), \\
35(7), 36(5), 37(7), 56(13), 60(3), 62,74(4), 77(2), 80,88(6), \\
90(11), 92(3), 93,99(2), 100(4), 102(6), 132(2), 192(4) *, 193(6), \\
194(2), 195(5), 196(5), 197(3), 198(13), 199(15), 200(5), 201(6), \\
202(7), 203(6), 204(6), 205(7), 206(29), 207(7), 209(8), 210(52), \\
211(3), 212(3), 213(12), 214(17), 215(16), 216(12), 217(19), \\
218(9), 219(56), 220(44), 221(18), 222(26), 223(68), 224(12), \\
225(13), 226(15), 227(58), 228(19), 229(22), 230(11), 231(5), \\
232(69), 233(46), 234(38), 235(23), 236(20), 237(33), 238(9), \\
239(5), 240(18), 241(27), 242(15), 243(24), 244(31), 260^{*}, 261, \\
262(4), 263,264(5), 265,267^{*}\end{array}$ \\
\hline 78 & $\begin{array}{ccc}28 / 24 & 0 / 0 & 28 / 24 \\
69(2), 70,73,74,77,78,79,81,82,87, \\
208,263\end{array}$ \\
\hline
\end{tabular}


Table 1: $(16,4,16,4)$ RDS with $N=\mathbb{Z}_{2} \times \mathbb{Z}_{2}$

\begin{tabular}{|c|c|c|c|c|}
\hline \# & Order & 2-Rank & Abelian & Nonabelian \\
\hline 99 & $\begin{array}{l}1152 \\
60(3), 267^{*}\end{array}$ & 20 & $4 / 2$ & $3 / 1$ \\
\hline 100 & $\begin{array}{l}1152 \\
60(3), 267^{*}\end{array}$ & 22 & $4 / 2$ & $3 / 1$ \\
\hline 103 & $\begin{array}{l}384 \\
60(3), 267^{*}\end{array}$ & 20 & $4 / 2$ & $3 / 1$ \\
\hline 104 & $\begin{array}{l}384 \\
60(3), 267^{*}\end{array}$ & 22 & $4 / 2$ & $3 / 1$ \\
\hline 105 & $\begin{array}{l}1152 \\
60(3), 267^{*}\end{array}$ & 18 & $4 / 2$ & $3 / 1$ \\
\hline 107 & $\begin{array}{l}6912 \\
60(3), 193,\end{array}$ & $\begin{array}{l}20 \\
67^{*}\end{array}$ & $5 / 3$ & $4 / 2$ \\
\hline 109 & $\begin{array}{l}384 \\
100,109,20\end{array}$ & $\begin{array}{l}22 \\
4,247\end{array}$ & $4 / 4$ & $4 / 4$ \\
\hline 111 & $\begin{array}{l}512 \\
20(2), 23(2) \\
92(2), 100, \\
\end{array}$ & $\begin{array}{l}20 \\
58(2), 6 \\
09,193(\end{array}$ & $\begin{array}{l}52 / 191 / 1 \\
), 69(6), 71(2), 74(3), \\
02(6), 204(2), 207(2),\end{array}$ & $\begin{array}{l}51 / 18 \\
3), 78(3), 90 \\
267^{*}\end{array}$ \\
\hline 113 & $\begin{array}{l}1536 \\
20(2), 23(2) \\
92,100,109\end{array}$ & $\begin{array}{l}20 \\
58(2), 6 \\
193(2),\end{array}$ & $\begin{array}{c}48 / 191 / 1 \\
), 69(6), 71(2), 74(3), \\
(3), 204(2), 207(2), 24\end{array}$ & $\begin{array}{l}47 / 18 \\
3), 78(3), 90 \\
67^{*}\end{array}$ \\
\hline 115 & $\begin{array}{l}256 \\
9(2), 132(2)\end{array}$ & 20 & $4 / 2$ & $4 / 2$ \\
\hline 116 & $\begin{array}{l}768 \\
9(2), 132(2)\end{array}$ & 20 & $4 / 2$ & $4 / 2$ \\
\hline 117 & 128 & 21 & $0 / 0$ & $0 / 0$ \\
\hline 120 & $\begin{array}{l}1536 \\
20(2), 23(2) \\
193,202(4),\end{array}$ & $\begin{array}{l}20 \\
58,60(6 \\
204,207\end{array}$ & $\begin{array}{lc}31 / 19 & 1 / 1 \\
(2), 71,74,75,78,90, \\
7,267^{*}\end{array}$ & $\begin{array}{l}30 / 18 \\
(2), 100,109,\end{array}$ \\
\hline 121 & $\begin{array}{l}384 \\
60(3), 267^{*}\end{array}$ & 22 & $4 / 2$ & $3 / 1$ \\
\hline 122 & $\begin{array}{l}128 \\
100,109,20\end{array}$ & $\begin{array}{l}22 \\
4,247\end{array}$ & $4 / 4$ & $4 / 4$ \\
\hline 123 & $\begin{array}{l}192 \\
20\end{array}$ & 22 & $1 / 1$ & $1 / 1$ \\
\hline 124 & $\begin{array}{l}64 \\
23\end{array}$ & 22 & $1 / 1$ & $1 / 1$ \\
\hline
\end{tabular}


Table 1: $(16,4,16,4)$ RDS with $N=\mathbb{Z}_{2} \times \mathbb{Z}_{2}$

\begin{tabular}{|c|c|c|c|c|c|}
\hline$\#$ & Order & 2-Rank & Total & Abelian & Nonabelian \\
\hline \multirow[t]{2}{*}{125} & 64 & 22 & $1 / 1$ & $0 / 0$ & $1 / 1$ \\
\hline & 132 & & & & \\
\hline \multirow[t]{2}{*}{126} & 192 & 22 & $1 / 1$ & $0 / 0$ & $1 / 1$ \\
\hline & 132 & & & & \\
\hline \multirow[t]{2}{*}{127} & 768 & 20 & $6 / 3$ & $1 / 1$ & $5 / 2$ \\
\hline & $60(4)$ & $267^{*}$ & & & \\
\hline \multirow[t]{2}{*}{128} & 256 & 20 & $7 / 3$ & $1 / 1$ & $6 / 2$ \\
\hline & $60(4)$ & ), $267^{*}$ & & & \\
\hline \multirow[t]{2}{*}{131} & 768 & 20 & $6 / 3$ & $1 / 1$ & $5 / 2$ \\
\hline & $60(4)$ & $267^{*}$ & & & \\
\hline 132 & 512 & 20 & $52 / 32$ & $1 / 1$ & $51 / 31$ \\
\hline
\end{tabular}

23, 24, 32, 33(2), 58, 59(2), 61, 62, 63, 65(2), 66, 67(3), 68(3), $69(7), 70,71,72,75,77(3), 78(2), 80,81(2), 85,90(2), 98(2)$, 101(2), 139, 192*, 197, 204, 205, 212

\begin{tabular}{|c|c|}
\hline 134 & $\begin{array}{l}64 / 21 \quad 0 / 0 \\
(2), 69(10), 70(5), 71(4) \\
3), 81(2), 195(2), 196,\end{array}$ \\
\hline 136 & $\begin{array}{l}512 \quad 20 \\
23,24,32,35(2), 55^{*}, 58(2), 59,61,63(3), 66(2), 67(4), 69(8), \\
71,72(4), 74(3), 75,76,78(2), 81(2), 90(2), 98,101(2), 104,136, \\
197,208,255,262\end{array}$ \\
\hline 137 & $\begin{array}{lcccc}768 & 20 & 24 / 13 & 0 / 0 & 24 / 13 \\
57,59,69(4), 70(3), 72,77(3), 78(2), 79(2), 80(3), 81,82,197, & \\
212 & & & \end{array}$ \\
\hline & $45 / 3$ \\
\hline
\end{tabular}
23, 24, 32(2), 33(4), 34(4), 55*, 58, 61, 62(2), 65, 67, 69, 70, 71, 75, 77, 79, 90(2), 98, 101(2), 104, 134(2), 139(3), 194, 196, 203, $205(2), 209,241(2), 254$

$\begin{array}{llllll}139 & 512 & 21 & 52 / 40 & 0 / 0 & 52 / 40\end{array}$

18, 20, 25(2), 89, 91(2), 92, 95, 97, 98, 100(3), 102(2), 105, 112, $115,117,119,120,129,131(2), 132,133(2), 145(2), 148,151$, 159, 160, 163, 164, 165, 166, 169(2), 198, 200, 207(2), 217(2), $227(2), 229,252,255,264$

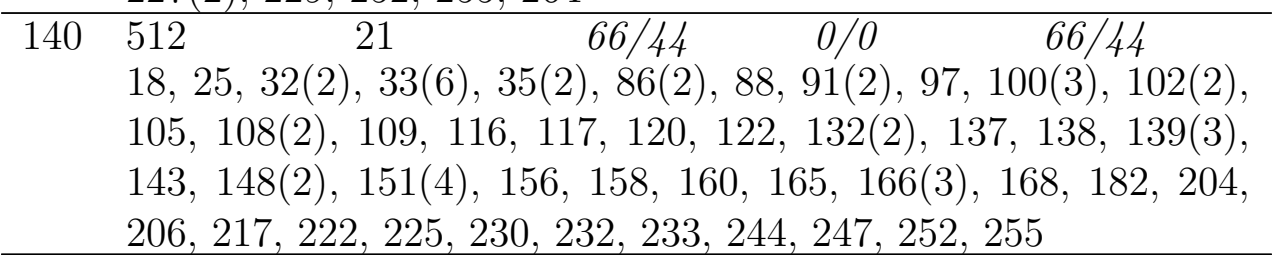


Table 1: $(16,4,16,4)$ RDS with $N=\mathbb{Z}_{2} \times \mathbb{Z}_{2}$

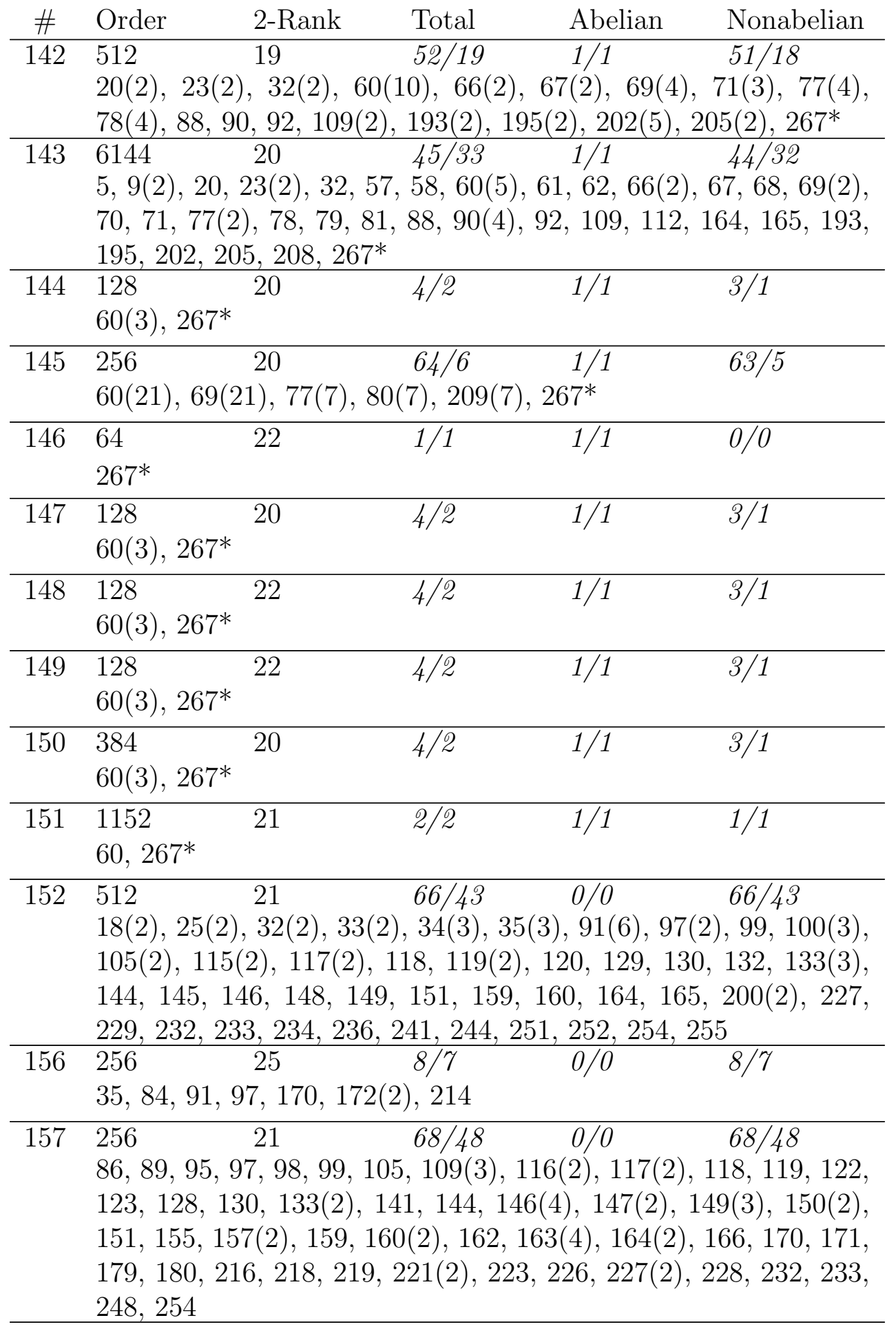


Table 1: $(16,4,16,4)$ RDS with $N=\mathbb{Z}_{2} \times \mathbb{Z}_{2}$

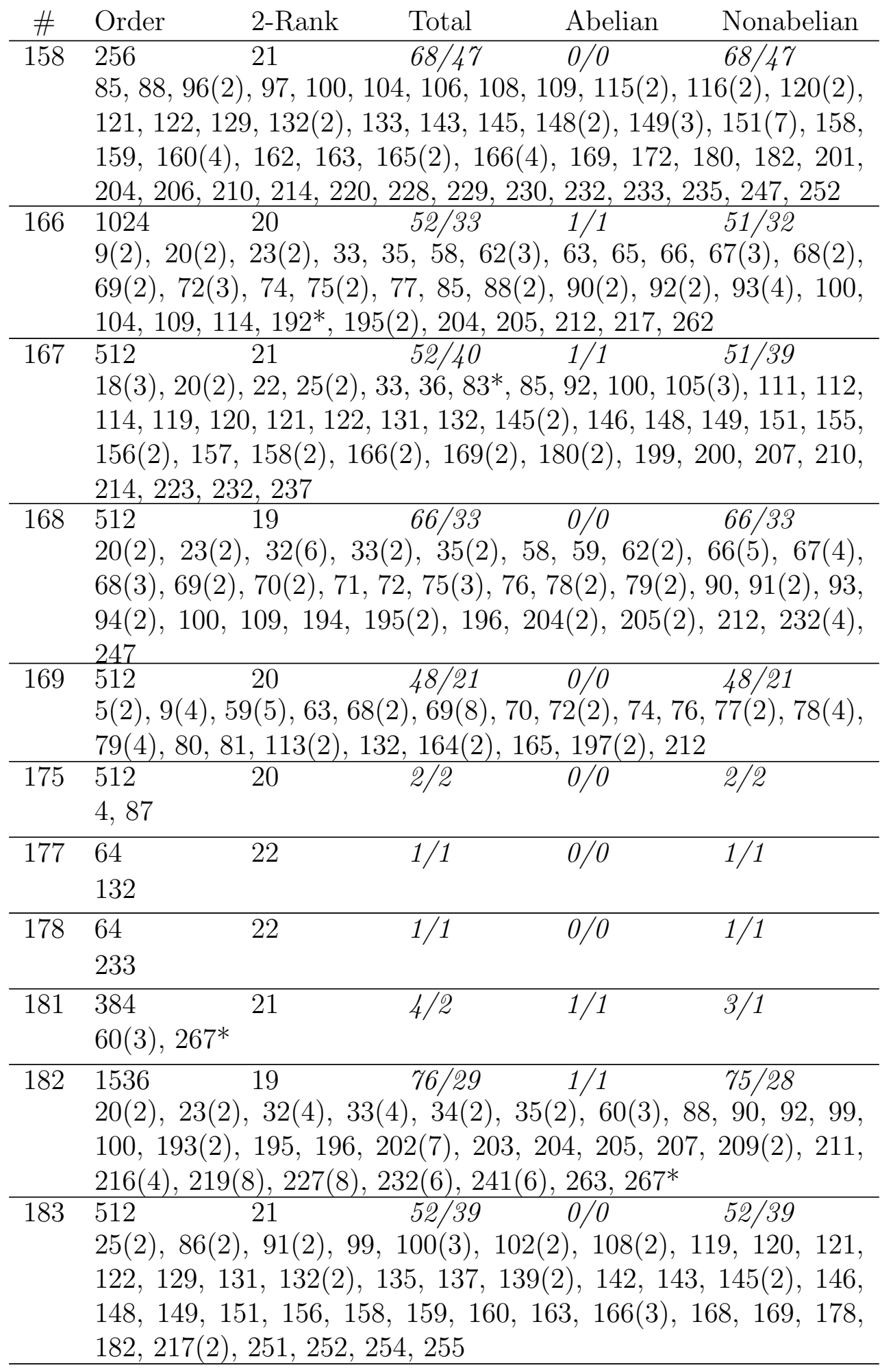


Table 1: $(16,4,16,4)$ RDS with $N=\mathbb{Z}_{2} \times \mathbb{Z}_{2}$

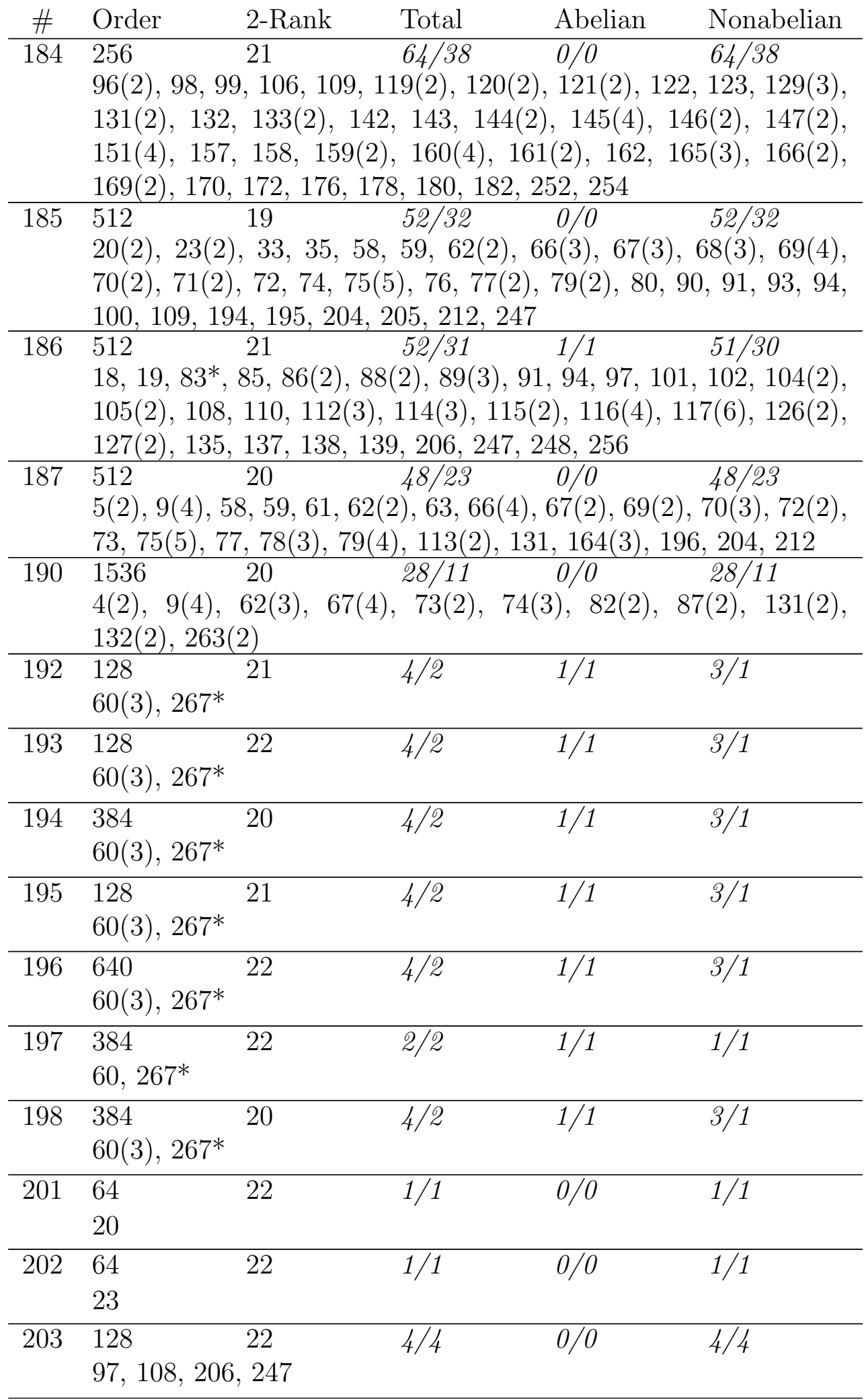


Table 1: $(16,4,16,4)$ RDS with $N=\mathbb{Z}_{2} \times \mathbb{Z}_{2}$

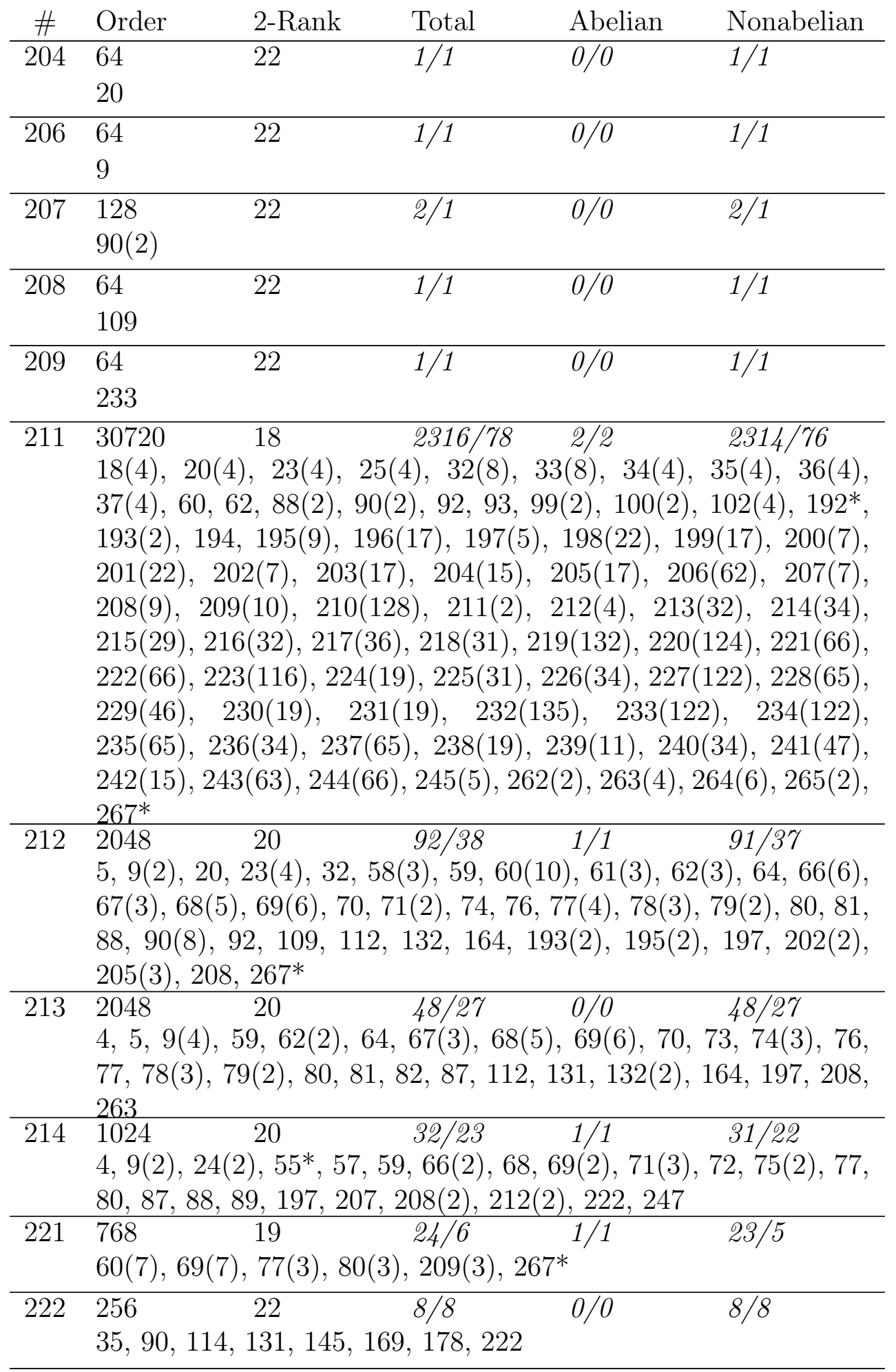


Table 1: $(16,4,16,4)$ RDS with $N=\mathbb{Z}_{2} \times \mathbb{Z}_{2}$

\begin{tabular}{cllccl}
$\#$ & Order & 2-Rank & Total & Abelian & Nonabelian \\
\hline 223 & 192 & 22 & $1 / 1$ & $0 / 0$ & $1 / 1$ \\
& 20 & & & & \\
\hline 224 & 768 & 20 & $24 / 6$ & $1 / 1$ & $23 / 5$ \\
& $60(7), 69(7), 77(3), 80(3), 209(3), 267^{*}$ & \\
\hline 225 & 256 & 20 & $64 / 6$ & $1 / 1$ & $63 / 5$ \\
& $60(21), 69(21), 77(7), 80(7), 209(7), 267^{*}$ & \\
\hline 226 & 320 & 21 & $1 / 1$ & $1 / 1$ & $0 / 0$ \\
& $267^{*}$ & & & & \\
\hline
\end{tabular}

Table 2: $(16,4,16,4)$ RDS with $N=\mathbb{Z}_{4}$

\begin{tabular}{clllll}
$\#$ & Order & 2-Rank & Total & Abelian & Nonabelian \\
\hline 1 & 512 & 22 & $14 / 9$ & $0 / 0$ & $14 / 9$
\end{tabular}
$58,59(2), 61(2), 72(4), 85,175,208,212,262$

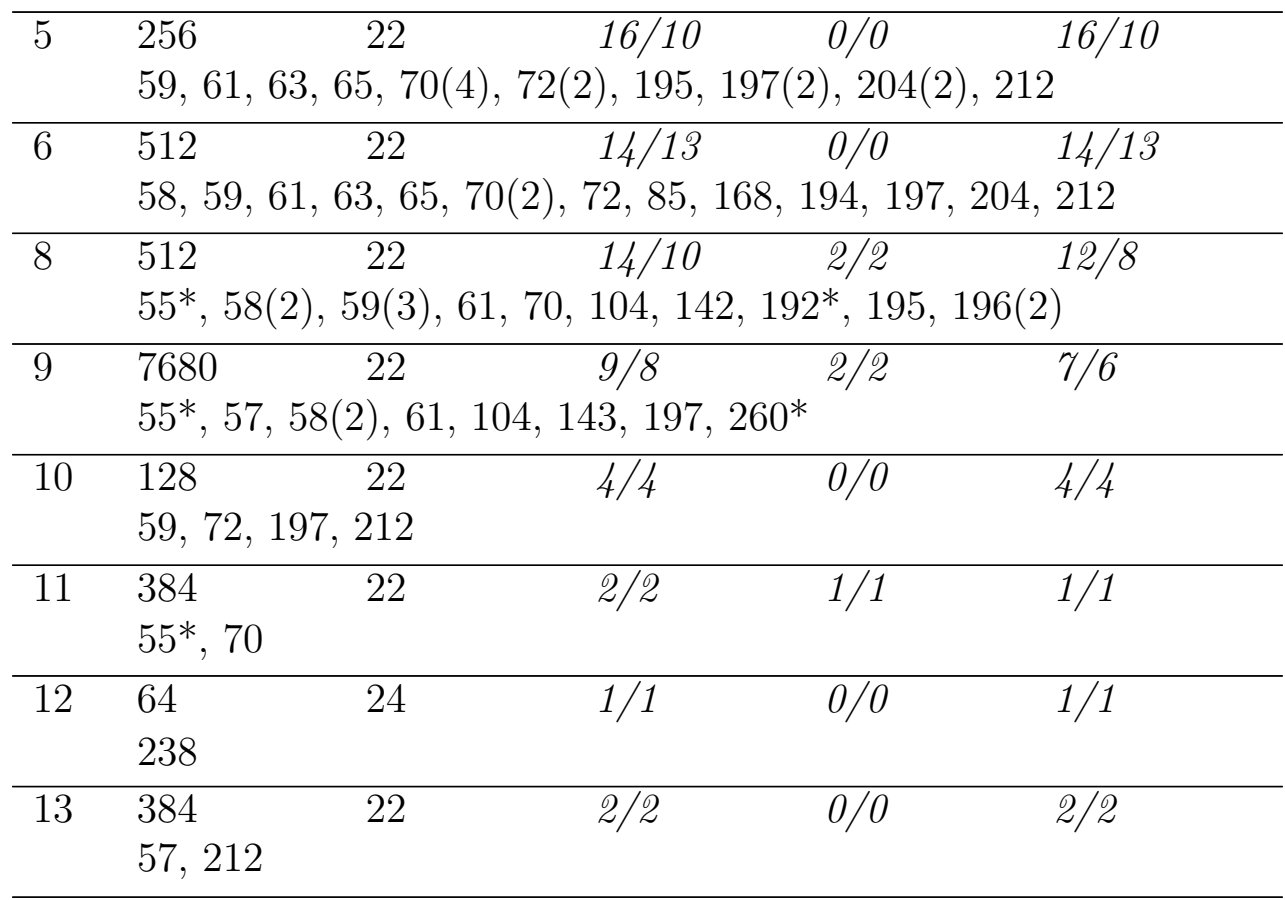


Table 3: Structures of abelian regular subgroups in $\mathbb{Z}_{2} \times$ $\mathbb{Z}_{2}$ nets

\begin{tabular}{|r|l|l|l|}
$\#$ & Order & Abelian & Group structure \\
\hline 1 & 1105920 & $6 / 2$ & $\mathbb{Z}_{2}^{2} \times \mathbb{Z}_{4}^{2}(4), \mathbb{Z}_{2}^{6}(2)$ \\
\hline 2 & 13824 & 2/2 & $\mathbb{Z}_{2}^{2} \times \mathbb{Z}_{4}^{2}, \mathbb{Z}_{2}^{6}$ \\
\hline 5 & 18432 & $6 / 2$ & $\mathbb{Z}_{2}^{2} \times \mathbb{Z}_{4}^{2}(5), \mathbb{Z}_{2}^{6}$ \\
\hline 9 & 4608 & 2/2 & $\mathbb{Z}_{2}^{2} \times \mathbb{Z}_{4}^{2}, \mathbb{Z}_{2}^{6}$ \\
\hline 22 & 6144 & $6 / 2$ & $\mathbb{Z}_{2}^{2} \times \mathbb{Z}_{4}^{2}(5), \mathbb{Z}_{2}^{4} \times \mathbb{Z}_{4}$ \\
\hline 31 & 1536 & $1 / 1$ & $\mathbb{Z}_{2}^{4} \times \mathbb{Z}_{4}$ \\
\hline 32 & 128 & $1 / 1$ & $\mathbb{Z}_{2}^{3} \times \mathbb{Z}_{8}$ \\
\hline 33 & 512 & $1 / 1$ & $\mathbb{Z}_{2}^{3} \times \mathbb{Z}_{8}$ \\
\hline 36 & 73728 & $6 / 3$ & $\mathbb{Z}_{2}^{2} \times \mathbb{Z}_{4}^{2}(4), \mathbb{Z}_{2}^{4} \times \mathbb{Z}_{4}, \mathbb{Z}_{2}^{6}$ \\
\hline 132 & 512 & $1 / 1$ & $\mathbb{Z}_{2}^{2} \times \mathbb{Z}_{4}^{2}$ \\
\hline 136 & 512 & $1 / 1$ & $\mathbb{Z}_{4}^{3}$ \\
\hline 138 & 1536 & $1 / 1$ & $\mathbb{Z}_{4}^{3}$ \\
\hline 166 & 1024 & $1 / 1$ & $\mathbb{Z}_{2}^{2} \times \mathbb{Z}_{4}^{2}$ \\
\hline 167 & 512 & $1 / 1$ & $\mathbb{Z}_{2} \times \mathbb{Z}_{4} \times \mathbb{Z}_{8}$ \\
\hline 186 & 512 & $1 / 1$ & $\mathbb{Z}_{2} \times \mathbb{Z}_{4} \times \mathbb{Z}_{8}$ \\
\hline 211 & 30720 & $2 / 2$ & $\mathbb{Z}_{2}^{2} \times \mathbb{Z}_{4}^{2}, \mathbb{Z}_{2}^{6}$ \\
\hline 214 & 1024 & $1 / 1$ & $\mathbb{Z}_{4}^{3}$ \\
\hline
\end{tabular}

Table 4: Structures of abelian regular subgroups in $\mathbb{Z}_{4}$ nets.

\begin{tabular}{|r|l|l|l|}
$\#$ & Order & Abelian & Group structure \\
\hline 8 & 512 & $2 / 2$ & $\mathbb{Z}_{4}^{3}, \mathbb{Z}_{2}^{2} \times \mathbb{Z}_{4}^{2}$ \\
\hline 9 & 7680 & $2 / 2$ & $\mathbb{Z}_{4}^{3}, \mathbb{Z}_{2}^{4} \times \mathbb{Z}_{4}$ \\
\hline 11 & 384 & $1 / 1$ & $\mathbb{Z}_{4}^{3}$ \\
\hline
\end{tabular}

Table 5: All regular abelian subgroups of order 64 appearing in GDDs with bitranslation group $\mathbb{Z}_{4}$.

\begin{tabular}{ll} 
Group & Structure \\
\hline 55 & $\mathbb{Z}_{4}^{3}$ \\
192 & $\mathbb{Z}_{2}^{2} \times \mathbb{Z}_{4}^{2}$ \\
260 & $\mathbb{Z}_{2}^{4} \times \mathbb{Z}_{4}$
\end{tabular}

Table 6: All regular abelian subgroups of order 64 appearing in GDDs with bi-translation group $\mathbb{Z}_{2} \times \mathbb{Z}_{2}$. 


\begin{tabular}{ll} 
Group & Structure \\
\hline 55 & $\mathbb{Z}_{4}^{3}$ \\
83 & $\mathbb{Z}_{2} \times \mathbb{Z}_{4} \times \mathbb{Z}_{8}$ \\
192 & $\mathbb{Z}_{2}^{2} \times \mathbb{Z}_{4}^{2}$ \\
246 & $\mathbb{Z}_{2}^{3} \times \mathbb{Z}_{8}$ \\
260 & $\mathbb{Z}_{2}^{4} \times \mathbb{Z}_{4}$ \\
267 & $\mathbb{Z}_{2}^{6}$
\end{tabular}

Table 7: Abelian groups which do not contain any $(16,4,16,4)$ RDS

\begin{tabular}{l|l} 
Group & Structure \\
\hline 1 & $\mathbb{Z}_{64}$ \\
2 & $\mathbb{Z}_{8} \times \mathbb{Z}_{8}$ \\
26 & $\mathbb{Z}_{4} \times \mathbb{Z}_{16}$ \\
50 & $\mathbb{Z}_{2} \times \mathbb{Z}_{32}$ \\
183 & $\mathbb{Z}_{2}^{2} \times \mathbb{Z}_{16}$
\end{tabular}

\section{Acknowledgments}

The authors wish to thank the referees for their useful comments. This research was partially supported by NSA Grant H98230-12-0213.

\section{References}

[1] Beth, T., Jungnickel, D., Lenz, H.: Design Theory, 2nd edition. Cambridge University Press, Cambridge (1999).

[2] Bosma, W., Cannon, J.: Handbook of Magma Functions, Department of Mathematics, University of Sydney, 1994.

[3] Gibbons, P.B., Mathon, R.: Enumeration of generalized Hadamard matrices of order 16 and related designs. J. Combin. Des. 17 (2009), 119-135.

[4] Harada, M., Lam, C., Tonchev, V.D.: Symmetric $(4,4)$-nets and generalized Hadamard matrices over groups of order 4, Des. Codes Cryptogr., 34 (2005), 71-87.

[5] Pott, A.: A survey on relative difference sets, in: Groups, Difference Sets, and the Monster, Arasu, K.T., Dillon, J.F., Harada, K., Seghal, S.K,, and Solomon, R.I., eds., DeGruyter Verlag, Berlin 1996, pp. 195-233.

[6] Röder, M.: The quasiregular projective planes of order 16, Glasnik Mat., 43 (2008), 231-242.

[7] Schmidt, B.: On $\left(p^{a}, p^{b}, p^{a}, p^{a-b}\right)$-Relative Difference Sets, J. Algebraic. Combin. 6 (1997), 279-297. 